\title{
Elisabeth Hildt \\ Electrodes in the brain: Some anthropological and ethical aspects of deep brain stimulation
}

\begin{abstract}
:
In the following text, medical, anthropological and ethical issues of deep brain stimulation, a medical technology in which electrodes implanted in the human brain electrically influence specified brain regions, will be discussed. After a brief account of the deep brain stimulation procedure and its chances and risks, anthropological and ethical aspects of the approach will be discussed. These relate to the reversibility of the procedure and to the patient's capacity to control the effects it exerts in the brain, to modifications and fluctuations in a person's character traits and individuality brought about by neurostimulation, and to the range of legitimate, adequate uses of the deep brain stimulation approach. The paper concludes that deep brain stimulation should be confined to therapeutic contexts and to severe, otherwise treatment-refractory disorders in which the aim is to normalize brain functioning. Apart from this, it should not be used to modify a person's individual character traits and behaviour or to enhance human traits.
\end{abstract}

\section{Agenda}

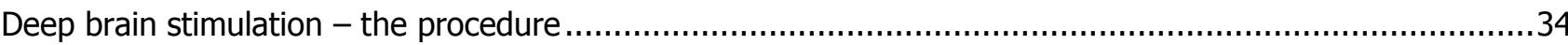

Direct interaction of man, brain and machine - some anthropological and ethical aspects ........................36

What about the capacity to control the effects of neurostimulation? ..............................................36

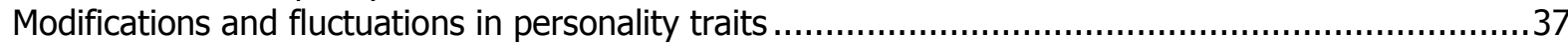

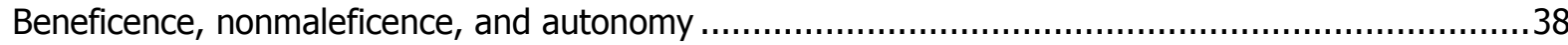

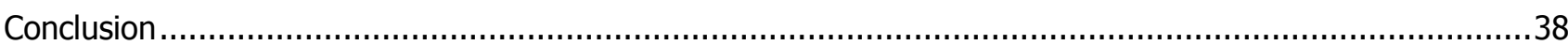

\section{Author:}

PD. Dr. Elisabeth Hildt:

Chair for Ethics in the Life Sciences, University of Tübingen, Wilhelmstr. 19, 72074 Tübingen, Germany

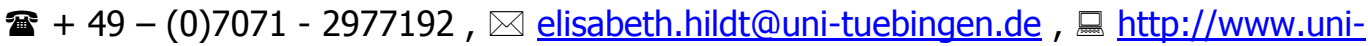

tuebingen.de/bioethik/index.htm 
During the past few years, our knowledge of the structure and function of the human brain has increased considerably, and this has prompted the development and use of new therapeutic strategies, among them technological procedures such as Information and Communication Technology implants (ICT implants) for the human brain. ${ }^{1}$ These include direct brain computer interfaces (BCIs) which allow brain signals to be used for communication and control of movement. Apart from noninvasive approaches such as the so-called thought translation device, which is a biofeedback communication system used by locked-in patients, invasive brain computer interfaces are currently being developed which aim at enabling the brain to exert direct motor control. In animal experiments using microelectrode arrays implanted in the motor cortex, it has been possible to control movements merely by "thinking them through." A related research strategy aims at developing implantable brain chips called "artificial hippocampus" that in several years' time might serve to restore memory in patients with Alzheimer's disease or stroke and that may even be used to enhance memory in "normal persons."

Another field which has drawn enormous clinical interest of late is the use of deep brain stimulation techniques in which electrodes implanted in the human brain electrically influence specified brain regions. Until now, this promising technology has mainly been used in the treatment of Parkinson's disease and other movement disorders. Apart from its successful application as a therapeutic approach to treating Parkinson's disease, an intense and controversial discussion is currently being conducted as to whether or not neurostimulation should be used in particular cases of severe and treatmentrefractory neuropsychiatric disorders such as obsessive-compulsive disorder (OCD).

The deep brain stimulation (DBS) procedure raises enormous hopes for individual patients suffering from severe diseases. However, the approach also involves considerable risks, and in light of the fact that it exerts a direct technological influence on the human brain, this being the organ which determines, like no other, a person's entire physical and mental existence, it poses complex anthropological and ethical questions. Concerning the individual patient, aspects to be discussed include possible benefits and risks of the procedure, especially issues that relate to claims that it is reversible and that the

1 cf. Hildt 2005 consequences of DBS can be controlled. Possible modifications in a patient's character traits brought about as a result of the procedure are also an issue of critical inquiry. More general issues subject to anthropological and ethical reflection relate to implications which the direct interplay between man, brain and technical devices might have for our conceptions of human identity and authenticity.

In the following, medical, anthropological and ethical issues of neurostimulation will be discussed, starting with a brief account of the deep brain stimulation procedure.

\section{Deep brain stimulation - the procedure}

Until now, deep brain stimulation has mainly been used in patients with idiopathic Parkinson's disease, a slowly progressing neurodegenerative disorder characterized by resting tremor, bradykinesia, muscular rigidity and gait disturbances. Neurostimulation aims at providing symptomatic relief in patients with Parkinson's disease and other movement disorders who are medically refractory to standard treatments. ${ }^{2}$

In this new and promising technology, an electrode lead with four electrode contacts is implanted bilaterally in the subthalamic nucleus under stereotactic conditions. The electrode lead is connected via wires which run subcutaneously to a battery-driven, programmable pulse generator placed below the clavicle. The implantation of the electrode is performed while the patient is awake to allow testing for optimal electrode placement and exclusion of adverse effects using microelectrode stimulation. After the implantation has been completed, the pulse generator is programmed to adjust the electrode system to the individual patient's needs by specifying which electrode contacts are to be used and by optimizing the various stimulation parameters such as pulse width, frequency and amplitude of stimulation. The DBS approach calls for intense post-operative care which involves multiple sessions for device adjustments and medication management. $^{3}$

${ }^{2}$ Okun \& Foote 2004; Rosenow et al. 2004; Benabid et al. 2005; Gharabaghi et al. 2005

${ }^{3}$ Andrews 2003 
By effecting chronic bilateral stimulation of the subthalamic nucleus in patients with Parkinson's disease, considerable improvement of motor symptoms and a reduction in medication dosages can be achieved. Best results have been obtained in patients with idiopathic Parkinson's disease who show no cognitive difficulties and who have preserved a good response to L-Dopa but manifest side-effects due to long-term L-Dopa treatment. ${ }^{4}$ The scientific community has no good understanding of the mechanism which underlies the positive effect of DBS in Parkinson's disease, however. ${ }^{5}$

The surgery also involves significant risks. These include intracranial hemorrhage in cases where blood vessels are punctured by microelectrodes or the DBS lead, which may cause strokes or strokelike symptoms, infection, various neurological problems, depression, memory problems and psychiatric and/or behavioural symptoms. Other problems can arise if the lead migrates or an electrode, connecting wire or impulse generator needs to be replaced. ${ }^{6}$

In addition to excellent motor benefits brought about by DBS in patients with Parkinson's disease, various negative neuropsychological consequences of the procedure have been reported. Although DBS usually involves no global cognitive or intellectual deterioration, mild cognitive impairments such as diminished verbal fluency and/or behavioural changes that seem to be comparatively rare and mostly transient have been observed. In individual cases, pronounced modifications in mood and behaviour have been reported, however. ${ }^{7}$ In particular, elderly patients with advanced Parkinson's disease are at risk for cognitive impairment. Neuropsychological changes observed in these patients include significant declines in working memory, speed of mental processing, bimanual motor speed and co-ordination, set switching, and declines in verbal learning, visual learning and memory. In addition, changes in mood and personality, especially frontal-lobe-related personality changes, have

4 Saint-Cyr et al. 2000; Okun \& Foote 2004; Funkiewiez et al. 2004; Rosenow et al. 2004; Benabid et al. 2005

${ }^{5}$ Garcia et al. 2005

6 Andrews 2003; Umemura et al. 2003; Okun \& Foote 2004; Rosenow et al. 2004

${ }^{7}$ Funkiewiez et al. 2004 been observed. ${ }^{8}$ At present it is not known whether the neuropsychological effects of DBS are reversible or not. ${ }^{9}$

Several clinical studies are currently investigating whether DBS might have a positive effect on treatment-refractory cases of neuropsychiatric disorders such as obsessive-compulsive disorder. ${ }^{10}$ Patients with obsessive-compulsive disorder (OCD) are tormented by obsessions which dominate their lives and impose inflexible rules on them. Although hypotheses have been made concerning dysfunctions in neural circuits connecting the frontal lobe and the basal ganglia (fronto-striatal-pallidalthalamic-frontal loops), the neurobiological basis of OCD is not well understood.

When used for OCD, DBS entails stimulation of the anterior limbs of the internal capsules or the ventral caudate nucleus. Until now, this strategy has been used in a very limited number of patients with severe, chronic, treatment-refractory OCD. In most of the patients studied so far, a positive effect of DBS on psychopathology and neuropsychological functioning has been reported. In most of the studies, the procedure has evidenced no deleterious impact or any major adverse or harmful consequences on the personality of the patients involved. ${ }^{11}$ In one case, a patient is reported to have suffered from DBS-induced panic and anxiety. ${ }^{12}$

Other uses of the neurostimulation technology include hypothalamic deep brain stimulation as a treatment for intractable chronic cluster headache and vagus nerve stimulation in epileptic patients with treatment-refractory partial-onset seizures. ${ }^{13}$ In addition, vagus nerve stimulation is currently under investigation in patients with major depressive

${ }^{8}$ Saint-Cyr et al. 2000; Funkiewiez et al. 2004

${ }^{9}$ Saint-Cyr et al. 2000; Bejerot 2003

10 Gabriëls et al. 2003; Aquizerate et al. 2004; Rauch et al. 2006

11 Gabriëls et al. 2003; Aquizerate et al. 2004; Cosgrove 2004

${ }^{12}$ Shapira et al. 2006

${ }^{13}$ Andrews 2003; Schoenen et al. 2005 
episodes ${ }^{14}$ in order to find out whether there is an anti-depressant effect of vagus nerve stimulation.

\section{Direct interaction of man, brain and machine - some anthropological and ethical aspects}

Without a doubt, computers and other technical devices are part and parcel of modern everyday life. Technical instruments also constitute a crucial dimension of modern medicine. Recent developments in neurotechnology which involve direct interactions between man and machine clearly have to be discussed against this background.

As opposed to the customary, traditional use of tools and technical instruments such as drills, computers and MP3 players and in contrast to external medical devices such as eyeglasses and hearing aids, neurotechnological instruments used for neurostimulation and other purposes are technical tools which enter the human body and affect it directly. In deep brain stimulation, an electrode is implanted in a specified region of the human brain, with subcutaneous wires connecting the electrode to an impulse generator implanted below the clavicle. The medical use of this technology has been facilitated by the availability of small high-performance computers and other small, biocompatible devices.

Although analogies can undoubtedly be drawn between the normal use of external tools, computers and other technical instruments and the use of "tools" incorporated into the human body, a crucial distinction must be made here: whenever a "tool" is integrated into the human body - especially into the part of the body which is so central to an individual's personality and overall existence, i.e., the brain - the distinction normally drawn between tools and the subject who use them is blurred. What is particularly important in this context is the fact that in contrast to modifications of other parts of the human body, modifications of the brain effected by direct integration of technical tools might subject the persons involved to alterations of individual traits.

In the following, implications of DBS resulting from the direct integration of technical tools into the human brain will be discussed.

\section{What about the capacity to control the effects of neurostimulation?}

An aspect of enormous importance relates to the question as to whether and/or to what degree it is possible to control the effects of electrodes on a patient's brain, i.e. as to what influences electrodes have on a patient's physical and mental traits. In this context, issues concerning the reversibility or irreversibility of the effects brought about by the treatment play an important role, as does the individual's ability to exercise control over these influences.

Clearly, DBS is a form of therapy less reversible than pharmacological forms of treatment in which adverse or unforeseen effects of medication can be modified without undue hardship - although even pharmacological treatment modalities may not be totally reversible on a molecular level. Nevertheless, DBS is quite a flexible procedure compared to classical surgical approaches. Unlike traditional psychosurgery which aims at leasioning a specified brain region ${ }^{15}$, neurostimulation only aims at exerting an electrical influence on the brain.

In neurostimulation, it is principally possible to adjust the electrode system to the individual patient. By programming the pulse generator, it is possible to modify the stimulation protocol and to adjust stimulation in accordance with the individual needs of the patient, the course of disease or other necessary modifications. One should not ignore the fact, however, that adjusting such devices is a complex, time-consuming procedure with manifold limitations. It may not be possible to achieve optimal therapeutic success, electrode stimulation may cause certain negative effects, etc. To be sure, in cases of severe and long-lasting problems, one does have the option of switching off the electrode or explanting the whole system.

Compared to less invasive approaches, neurostimulation is clearly not a completely reversible procedure, however. DBS involves stereotactic surgery, which entails structural modifications of the brain albeit limited ones - caused by macroelectrode implantation as well as manifold risks for brain lesions. Currently no one knows how far neurostimulation involves adverse, irreversible, longterm effects resulting from chronic stimulation. In particular, it remains to be investigated whether the

\footnotetext{
${ }^{15}$ cf. Bejerot 2003
}

${ }^{14}$ Nahas et al. 2005 
negative neuropsychological effects observed after DBS are reversible or not. ${ }^{16}$

Adverse effects, especially if they are irreversible, may not only modify a person's individuality, but also significantly reduce her well-being and autonomy. In sum, a person using DBS is strongly dependent on the electrode system. In order to allow the patient to control his overall situation as far as possible, it is necessary to have a specialist team available 24 hours a day and 7 days a week which is able to adjust, repair and (re)programme the system if problems, malfunction or deviations arise. The person's quality of life, her motor performance, her general mood and her overall psychical situation may depend to a high degree on how well the technical device functions. Disturbances, problems and malfunctioning of the electrode system have a direct impact on the individual.

\section{Modifications and fluctuations in personality traits}

In evaluating various neurostimulation procedures, the possibility that they might effect modifications in a person's behaviour, mental characteristics and personality plays an important role. On the one hand, psychomodulations or modifications in behaviour may be inherent to the therapeutic procedure; on the other hand, they may develop unintentionally in the form of unwanted or unexpected side-effects. Such psychomodulations pose manifold medical, philosophical and ethical questions as regards the personality, personal identity, individual responsibility, autonomy, authenticity and self-perception of the person involved. ${ }^{17}$ They also have complex implications for a person's individual, familial and social life.

Unlike other forms of therapy, deep brain stimulation involves direct modifications of a person's characteristics which can be brought about almost instantaneously. The person's "mode of existence" might be dependent on whether the electrode is activated or not. The following examples serve to illustrate this capacity of DBS to abruptly alter personality traits. In their publication on DBS in patients with OCD, Gabriëls et al. ${ }^{18}$ describe the

${ }^{16}$ Saint-Cyr et al. 2000, Bejerot 2003

${ }^{17}$ cf. Hildt 1999

${ }^{18}$ Gabriëls et al. 2003, 279 case of a woman who showed positive results after successful DBS. They state that "when stimulation was switched off she almost instantaneously became anxious and in great distress again". Funkiewiez et al. report on a woman with Parkinson's disease characterized preoperatively by fluctuations in mood which manifested themselves in concurrence with her motor symptoms. After deep brain stimulation improved the symptoms significantly, the authors described the patient's situation as follows: ${ }^{19}$ "When stimulation was deactivated for motor evaluation, the patient immediately felt overwhelming sadness, dissolved in tears, and experienced a progressive reappearance of severe parkinsonism. When describing the impact of treatment, she said: 'If stimulation is switched off, I am dead; when on, I am alive. With medication on top, it's happiness and well being.' In the case of a woman with significant positive results after DBS for OCD, her husband stated: ${ }^{20}$ 'When stimulation is switched ON, she changes and improves suddenly, but we (he and her children) don't have a similar button that can be pressed to adapt at once to this new person."'

Undoubtedly these cases all show very good therapeutic success; they all involve considerable and highly welcome improvement of symptoms brought about by DBS. On the other hand, they underline the intensive and direct interaction between the patient and the electrode system and the extreme situation the patient finds himself in, being enormously dependent on stimulation. Motor performance, mood and personality may be perceived and interpreted as relying highly on the technical system, i.e. on an external component which exerts direct influence on the person's brain. This may lead to the impression of being - in one's essence manipulated by the electrode system.

In light of such fluctuations, questions concerning personal identity and the individuality of the patients involved may arise. In which condition is the person really "herself," the authentic person she considers herself to be? When the electrode is switched on or when it is switched off? Manifold difficulties in adapting to the different "modes of existence" may arise. Not only the patient involved but also the whole family has to cope with these abrupt changes in character traits that may occur when the elec-

${ }^{19}$ Funkiewiez et al. 2004, 837

${ }^{20}$ Gabriëls et al. 2003, 278 
trode is switched on or off or when there are variations in the stimulation protocol.

\section{Beneficence, nonmaleficence, and autonomy}

Deep brain stimulation is a promising technology that in principle can be used in manifold contexts. But which uses of the procedure can be considered adequate ones? In order to find an answer to that question, let me argue on the basis of the principlebased approach developed by Tom Beauchamp and James Childress. ${ }^{21}$

With regard to beneficence and nonmaleficence, the risk-benefit-ratio of the procedure is crucial. As discussed above, the surgical procedure bears considerable risks. Only those uses in which a therapeutic benefit might reasonably be expected and in which the (expected) benefits clearly outweigh the risks can be considered acceptable. That's why it should be confined to severe chronic disorders the pathophysiological basis of which is known. Otherwise, the hope for therapeutic benefits might prove to be pure speculation. In general, before DBS is chosen as a treatment by an individual patient, all other less invasive treatment options should have been taken into consideration.

Apart from these medical aspects: Is a person harmed by the incorporation of technical devices into the brain? Manifold concerns, fears and fantasies stem from the option to integrate computers and other technical instruments into the brain and other parts of the human body. These involve aspects such as the technicalization of the human body, the encouragement of a reductionist, technological view on human beings, the fear of losing human identity, and speculations relating to cyborgs (cybernetic organisms). ${ }^{22}$

Undoubtedly, all these concerns - which unequivocally reveal that there are ethical limits to the amount and range of human body parts to be substituted by technical devices - need broad and intense interdisciplinary discussion. However, the decisive criterion in DBS is not the fact that a technical device, i.e. an electrode, is being implanted into the human brain, but the question of whether the device is able to perform more or less the same function as the formerly intact, now degenerated or otherwise dysfunctional neurons do. From the point of view of functionalism, electrode stimulation merely serves to normalize brain function.

This points at another aspect of central relevance: the respect for autonomy of the person involved. In part, concerns and fears related to neurostimulation clearly originate from the perceived power of this technology to modify a person's character traits and individuality - and to do so even against that person's will. Also science-fiction ideas of persons being externally controlled by other persons or by computers may be seen against this background. So, for autonomy reasons neither the implantation of the electrode device nor any modification in the stimulation protocol should be done without having obtained the patient's free and informed consent. DBS should be confined to therapeutic contexts and to severe, otherwise treatment-refractory disorders in which the aim is to normalize brain functioning. Apart from this, it should not be used to modify a person's individual character traits and behaviour or to enhance human traits.

\section{Conclusion}

Deep brain stimulation is a new and promising approach. Especially in patients with Parkinson's disease who had been previously treatmentrefractory, significant improvements in symptoms have been brought about.

There are several problematic issues that call for further discussion, however. First of all, a detailed assessment of the clinical benefits and the adverse effects of this invasive procedure, especially on a long-term basis, is required. With regard to the individual patient involved, it is absolutely necessary to carefully analyze his situation, to evaluate the chances and risks of the DBS procedure and to seek the patient's free and informed consent. In neuropsychiatric disorders where judgement is impaired, manifold problems arise when it comes to attempting to obtain the patient's free and informed consent to this surgical procedure. ${ }^{23}$ Before undergoing DBS, all other less invasive options available should have been taken into consideration. This is especially true as concerns disorders such as OCD. As far as this is concerned, DBS is at present highly investigational and there is currently enormous uncertainty as to possible benefits and harms.

\footnotetext{
23 cf. Comité Consultatif National d'Ethique (CCNE)
2003

23 cf. Comité Consultatif National d'Ethique (CCNE)
2003
}

\footnotetext{
${ }^{21}$ Beauchamp \& Childress 2001

${ }^{22}$ Hildt 2005
} 
In view of the considerable risks and the inherent capacity of this technology to modify a person's character traits, the use of DBS should be restricted to severe disorders whose pathophysiological basis is well known and which are otherwise treatmentrefractory.

\section{References}

Andrews, R.J. (2003): Neuromodulation I. Techniques - Deep Brain Stimulation, Vagus Nerve Stimulation, and Transcranial Magnetic Stimulation, Ann. N.Y. Acad. Sci. 993: 1-13.

Aquizerate, B. et al. (2004): Deep brain stimulation of the ventral caudate nucleus in the treatment of obsessive-compulsive disorder and major depression, $J$ Neurosurg 101, 682-686.

Beauchamp, T.L. \& Childress, J.F. (2001): Principles of Biomedical Ethics, Oxford University Press: New York, Fifth Edition.

Bejerot, S. (2003): Editorial: Psychosurgery for obsessive-compulsive disorder - concerns remain, Acta Psychiatr Scand 107, 241-243.

Benabid, A.L. et al. (2005): Deep-brain stimulation in Parkinson's disease: long-term efficacy and safety - What happened this year?, Current Opinion in Neurology 18, 623-630.

Comité Consultatif National d'Ethique (CCNE) (2003): Functional Neurosurgery for Severe Psychiatric Disorders, Jahrbuch für Wissenschaft und Ethik 8, 531-547.

Cosgrove, G.R. (2004): Editorial: Deep brain stimulation and psychosurgery, J Neurosurg 101, 574576.

Funkiewiez, A. et al. (2004): Long term effects of bilateral subthalamic nucleus stimulation on cognitive function, mood, and behaviour in Parkinson's disease, J Neurol Neurosurg Psychiatry 75, 834-839.

Gabriëls, L. et al. (2003): Deep brain stimulation for treatment-refractory obsessive-compulsive disorder: psychopathological and neuropsychological outcome in three cases, Acta Psychiatr Scand 107, 275-282.

Garcia, L. et al. (2005): High-frequency stimulation in Parkinson's disease: more or less?, Trends in Neurosciences 28(4), 209-216.

Gharabaghi, A. et al. (2005): Wiederherstellung der Funktion: Modulation von Hirnfunktionen durch Neuroprothesen, in: Engels, E.-M. \& Hildt, E. (eds): Neurowissenschaften und Menschenbild, mentis: Paderborn, 57-75.
Hildt, E.: Ethical aspects of neural tissue transplantation, Croatian Medical Journal, Vol. 40, No. 3, 1999, 326-333.

Hildt, E.: Computer, Körper und Gehirn: Ethische Aspekte eines Wechselspiels, in Engels, E.-M. \& Hildt, E. (eds.): Neurowissenschaften und Menschenbild, mentis: Paderborn, 2005, 121-137.

Nahas, Z. et al. (2005): Two-Year Outcome of Vagus Nerve Stimulation (VNS) for Treatment of Major Depressive Episodes, J Clin Psychiatry 66, 1097-1104.

Okun, M.S. \& Foote, K.D. (2004): Deep Brain Stimulation - The $10 \mathrm{Key}$ Issues Every Practitioner, Patient and Family Member should be Familiar With, Business Briefing: North American Pharmacotherapy 2, 1-6, 2204.

Rauch, S.L. et al. (2006): A functional neuroimaging investigation of deep brain stimulation in patients with obsessive-compulsive disorder, J Neurosurg 104, 558-565.

Rosenow, J.M. et al. (2004): Deep brain stimulation for movement disorders, Neurological Research 26, 9-20.

Schoenen, J. et al. (2005): Hypothalamic stimulation in chronic cluster headache: a pilot study of efficacy and mode of action, Brain 128(4), 940-947.

Shapira, N.A. et al. (2006): Panic and fear induced by deep brain stimulation, Journal of Neurology, Neurosurgery and Psychiatry 77, 410-412.

Umemura, A. et al. (2003): Deep brain stimulation for movement disorders: morbidity and mortality in 109 patients, J Neurosurg 98, 779-784. 